\title{
Identification of black-box wave propagation models using large-scale convex optimization ${ }^{1}$
}

Toon van Waterschoot ${ }^{2}{ }^{3}$, Moritz Diehl ${ }^{2}$, Marc Moonen $^{2}$, and Geert Leus ${ }^{4}$

July 2012

Published in Preprints 16th IFAC Symp. System Identification (SYSID '12), Brussels, Belgium, July 2012, pp. 338-343 (invited paper).

\footnotetext{
${ }^{1}$ This report is available by anonymous ftp from ftp.esat.kuleuven.be in the directory pub/sista/vanwaterschoot/reports/11-209.pdf

${ }^{2}$ K.U.Leuven, Dept. of Electrical Engineering (ESAT), Research group SCD(SISTA), Kasteelpark Arenberg 10, 3001 Leuven, Belgium, Tel. +32 16321788 , Fax +32 16 321970, WWW: http://homes.esat.kuleuven.be/ tvanwate. E-mail: toon.vanwaterschoot@esat.kuleuven.be.

${ }^{3}$ This research was carried out at the ESAT laboratory of KU Leuven, and was supported by the KU Leuven Research Council (CoE EF/05/006 "Optimization in Engineering” (OPTEC), PFV/10/002 (OPTEC), IOF-SCORES4CHEM, Concerted Research Action GOA-MaNet), the Belgian Federal Science Policy Office (IUAP P6/04 "Dynamical systems, control and optimization" (DYSCO) 2007-2011), the Research Foundation Flanders - FWO (Postdoctoral Fellowship T. van Waterschoot, Research Projects G0226.06, G0321.06, G.0302.07, G.0320.08, G.0558.08, G.0557.08, G.0588.09, G.0600.08, Research Communities G.0377.09 and WOG: ICCoS, ANMMM, MLDM), the IWT (Research Projects Eureka-Flite+, SBO LeCoPro, SBO Climaqs, SBO POM, O\&O-Dsquare), the European Commission (Research Community ERNSI, Research Projects FP7-HD-MPC (INFSO-ICT- 223854), COST-intelliCIS, FP7-EMBOCON (ICT-248940), FP7-SADCO (MC ITN-264735), ERC HIGHWIND (259 166)), the NWO-STW (VICI project 10382), AMINAL, ACCM, and IBBT. The scientific responsibility is assumed by its authors.

${ }^{4}$ Delft University of Technology, Faculty of Electrical Engineering, Mathematics, and Computer Science, Mekelweg 4, 2628 CD Delft, The Netherlands, Tel. +31 15278 1372, Fax +31 15278 6190, WWW: http://cas.et.tudelft.nl. E-mail: g.leus@tudelft.nl.
} 


\title{
Identification of Black-Box Wave Propagation Models Using Large-Scale Convex Optimization
}

\author{
Toon van Waterschoot* Moritz Diehl* Marc Moonen* \\ Geert Leus** \\ * Department of Electrical Engineering (ESAT-SCD), Katholieke \\ Universiteit Leuven, 3001 Leuven, Belgium (e-mail: \\ \{tvanwate,mdiehl,moonen\}@esat.kuleuven.be) \\ ** Faculty of Electrical Engineering, Mathematics, and Computer \\ Science, Delft University of Technology, 2628 CD Delft, The \\ Netherlands (e-mail: g.j.t.leus@tudelft.nl)
}

\begin{abstract}
In this paper, we propose a novel approach to the identification of multiple-input multiple-output (MIMO) wave propagation models having a common-denominator pole-zero parametrization. We show how the traditional, purely data-based identification approach can be improved by incorporating a physical wave propagation model, in the form of a spatiotemporally discretized version of the wave equation. If the wave equation is discretized by means of the finite element method (FEM), a high-dimensional yet highly sparse linear set of equations is obtained that can be imposed at those frequencies where a high-resolution model estimate is desired. The proposed identification approach then consists in sequentially solving two largescale convex optimization problems: a sparse approximation problem for estimating the point source positions required in the FEM, and an equality-constrained quadratic program (QP) for estimating the common-denominator pole-zero model parameters. A simulation example for the case of indoor acoustic wave propagation is provided to illustrate the benefits of the proposed approach.
\end{abstract}

Keywords: Multivariable System Identification; Hybrid and Distributed System Identification; Vibration and Modal Analysis

\section{INTRODUCTION}

We consider wave propagation in a three-dimensional (3-D) enclosure with partially reflective boundaries as governed by the wave equation

$$
\nabla^{2} u(\mathbf{r}, t)-\frac{1}{c^{2}} \frac{\partial^{2}}{\partial t^{2}} u(\mathbf{r}, t)=s(\mathbf{r}, t)
$$

with appropriate boundary conditions in the spatiotemporal domain $\Omega \times T$. Here, $\mathbf{r}=[x, y, z] \in \Omega$ and $t \in T$ denote the spatial and temporal coordinates, respectively,

\footnotetext{
* This research was carried out at the ESAT laboratory of KU Leuven, and was supported by the KU Leuven Research Council ( $\mathrm{CoE}$ EF/05/006 "Optimization in Engineering" (OPTEC), PFV/10/002 (OPTEC), IOF-SCORES4CHEM, Concerted Research Action GOAMaNet), the Belgian Federal Science Policy Office (IUAP P6/04 "Dynamical systems, control and optimization" (DYSCO) 20072011), the Research Foundation Flanders - FWO (Postdoctoral Fellowship T. van Waterschoot, Research Projects G0226.06, G0321.06, G.0302.07, G.0320.08, G.0558.08, G.0557.08, G.0588.09, G.0600.08, Research Communities G.0377.09 and WOG: ICCoS, ANMMM, MLDM), the IWT (Research Projects Eureka-Flite+, SBO LeCoPro, SBO Climaqs, SBO POM, O\&O-Dsquare), the European Commission (Research Community ERNSI, Research Projects FP7-HDMPC (INFSO-ICT- 223854), COST-intelliCIS, FP7-EMBOCON (ICT-248940), FP7-SADCO (MC ITN-264735), ERC HIGHWIND (259 166)), the NWO-STW (VICI project 10382), AMINAL, ACCM, and IBBT. The scientific responsibility is assumed by its authors.
}

$s(\mathbf{r}, t)$ represents the driving source function that initiates the wave propagation, $u(\mathbf{r}, t)$ represents the resulting wave field, and $c$ is the wave propagation speed determined by the propagation mechanism and medium. If we consider the driving source function to be generated by $M$ point sources at positions $\mathbf{r}_{m}, m=1, \ldots, M$, then the wave field can be expressed as a superposition of $M$ contributions corresponding to the temporal convolution of the point source signals $s_{m}(t)$ with the Green's function $h\left(\mathbf{r}, \mathbf{r}_{m}, t\right)$,

$$
u(\mathbf{r}, t)=\sum_{m=1}^{M}\left(\int_{-\infty}^{\infty} s_{m}(\tau) h\left(\mathbf{r}, \mathbf{r}_{m}, t-\tau\right) d \tau\right) .
$$

If we observe the wave field at a discrete number of positions $\overline{\mathbf{r}}_{j}, j=1, \ldots, J$, it follows from (2) that the wave propagation can be modeled as a multiple-input multipleoutput (MIMO) linear time-invariant (LTI) system. If we assume the source signals $s_{m}(t)$ to be bandlimited, then the Green's function can be sampled in time and the MIMO-LTI system can be represented by a discrete-time transfer function matrix

$$
\begin{aligned}
\mathbf{H}(z) & =\sum_{n=0}^{\infty} \mathbf{H}_{n} z^{-n} \\
& =\sum_{n=0}^{\infty}\left[\begin{array}{ccc}
h\left(\overline{\mathbf{r}}_{1}, \mathbf{r}_{1}, n\right) & \ldots & h\left(\overline{\mathbf{r}}_{1}, \mathbf{r}_{M}, n\right) \\
\vdots & \ddots & \vdots \\
h\left(\overline{\mathbf{r}}_{J}, \mathbf{r}_{1}, n\right) & \ldots & h\left(\overline{\mathbf{r}}_{J}, \mathbf{r}_{M}, n\right)
\end{array}\right] z^{-n}
\end{aligned}
$$


where $n=t / T_{s}$ denotes the discrete time index, with $T_{s}$ the sampling period. It is particularly relevant to represent the transfer function matrix by means of a pole-zero model with a common denominator, i.e.,

$$
\mathbf{H}(z)=\frac{\sum_{n=0}^{Q} \mathbf{B}_{n} z^{-n}}{\sum_{n=0}^{P} a_{n} z^{-n}}
$$

with

$$
\mathbf{B}_{n}=\left[\begin{array}{ccc}
b_{n}\left(\overline{\mathbf{r}}_{1}, \mathbf{r}_{1}\right) & \ldots & b_{n}\left(\overline{\mathbf{r}}_{1}, \mathbf{r}_{M}\right) \\
\vdots & \ddots & \vdots \\
b_{n}\left(\overline{\mathbf{r}}_{J}, \mathbf{r}_{1}\right) & \ldots & b_{n}\left(\overline{\mathbf{r}}_{J}, \mathbf{r}_{M}\right)
\end{array}\right]
$$

Indeed, it was shown in Gustafsson et al. (2000) that such a parametrization is related to the "assumed modes solution" of the wave equation, in which the source and wave field are expanded on the eigenfunction basis of the enclosure, see also Kuttruff (2009). Here, the common denominator is related to the resonant modes of the enclosure which can be understood to be independent of the source and observer positions, see Gustafsson et al. (2000), Kuttruff (2009) and Haneda et al. (1994).

A parametric model of the wave propagation is particularly useful for the prediction, simulation, and deconvolution (i.e., source recovery) of the wave field, in case a fixed set of source and observer positions is considered. Even though these operations may also be performed through direct use of (a numerical approximation of) the wave equation in (1), the availability of a parametric model will typically result in significantly less computations. Because of the tight connection between the pole-zero model and the assumed modes solution of the wave equation, it may be appealing to use a grey-box model, by parametrizing the numerator and denominator in (5) explicitly as a function of the resonance frequencies and damping factors, see Gustafsson et al. (2000). However, this parametrization is highly nonlinear and so the parameter estimation requires the solution of a non-convex optimization problem. Therefore, we prefer to use a linear-in-the-parameters black-box pole-zero model.

Our aim is then to estimate the model parameters as accurately as possible, given a data set consisting of source and observed signal samples. A variety of estimation algorithms for identifying common-denominator pole-zero models has been proposed earlier in literature, see, e.g., Gustafsson et al. (2000), Haneda et al. (1994), Rolain et al. (1998), Stoica and Jansson (2000), Verboven et al. (2004), and Hikichi and Miyoshi (2004). A common property of these algorithms is that they rely exclusively on the available data set. Instead, we propose an approach in which not only the data set, but also the structure of the underlying wave equation is exploited in the estimation of the pole-zero model parameters. By enforcing the model parameters to obey a linear relationship derived from a finite element approximation of the wave equation, we can include physical arguments in the black-box identification while avoiding the non-convexity issues encountered with a grey-box approach. This allows to achieve a higher estimation accuracy as compared to the purely data-based algorithms in literature, or to achieve a similar accuracy with a smaller data set. The latter property is particularly appealing if the estimation of the common-denominator coefficients is of primary interest. The inclusion of the wave equation structure in the black-box identification problem can then result in a reduction of the number of observation positions required to achieve a given accuracy, which may significantly reduce the cost of the identification experiment.

The paper is organized as follows. In Section 2 we formulate the problem statement and review the existing data-based approach to the identification of commondenomimator pole-zero models. In Section 3, we show how the finite element method (FEM) can be used to derive a set of linear equations in the pole-zero model parameters, that are valid if the MIMO-LTI system is indeed governed by the wave equation in (1). This set of equations is then used in Section 4 to formulate a large-scale convex optimization problem that allows to identify the commondenominator pole-zero model by relying on both the data set and the wave equation structure. Finally, a simulation example is provided in Section 5.

\section{PROBLEM STATEMENT \& STATE OF THE ART}

\subsection{Problem Statement}

The problem considered in this paper can be formulated as follows. We are given a data set consisting of $N$ samples of the source signals and observed signals,

$$
Z^{N}=\left\{s_{m}(n), y_{j}(n)\right\}_{n=1}^{N}, m=1, \ldots, M, j=1, \ldots, J
$$

where the observed signals obey the measurement model

$$
y_{j}(n)=u\left(\overline{\mathbf{r}}_{j}, n\right)+v_{j}(n), j=1, \ldots, J .
$$

Here, the noise-free observations $u\left(\overline{\mathbf{r}}_{j}, n\right), j=1, \ldots, J$ result from a spatiotemporal sampling of the wave field $u(\mathbf{r}, t)$, as generated by the wave equation (1), and $v_{j}(n), j=1, \ldots, J$, represents measurement noise. For the sake of simplicity, and without loss of generality, we will assume that the measurement noise signals $v_{j}(n)$ are realizations of zero-mean and mutually uncorrelated white noise processes with equal variance $\sigma_{v}^{2}$. Our aim is then to obtain the best possible estimate of the parameter vector

$$
\boldsymbol{\theta}=\left[\begin{array}{lllll}
\mathbf{b}_{1}^{T} & \mathbf{b}_{2}^{T} & \ldots & \mathbf{b}_{M}^{T} & \mathbf{a}^{T}
\end{array}\right]^{T}
$$

containing the coefficients of the common-denominator pole-zero model in (5), with

$$
\begin{aligned}
& \mathbf{b}_{m}=\left[b_{0}\left(\overline{\mathbf{r}}_{1}, \mathbf{r}_{m}\right) \ldots b_{Q}\left(\overline{\mathbf{r}}_{1}, \mathbf{r}_{m}\right) \ldots b_{0}\left(\overline{\mathbf{r}}_{J}, \mathbf{r}_{m}\right) \ldots b_{Q}\left(\overline{\mathbf{r}}_{J}, \mathbf{r}_{m}\right)\right]^{T} \\
& \text { for } m=1, \ldots, M \text { and } \\
& \qquad \mathbf{a}=\left[a_{0} \ldots a_{P}\right]^{T} .
\end{aligned}
$$

Note that the first coefficient in the denominator parameter vector is usually fixed to $a_{0}=1$. We include it here in the parameter vector for notational convenience.

\subsection{State-of-the-Art Data-Based Identification Approach}

Different algorithms for the estimation of the parameter vector $\boldsymbol{\theta}$ using the data model (5)-(8) have been proposed, see Gustafsson et al. (2000), Haneda et al. (1994), Rolain et al. (1998), Stoica and Jansson (2000), Verboven et al. (2004), and Hikichi and Miyoshi (2004). In these algorithms, however, the knowledge that the noise-free observations $u\left(\overline{\mathbf{r}}_{j}, n\right), j=1, \ldots, J$, are samples of the wave field generated by (1) is not exploited, and hence the structure 


$$
\boldsymbol{\Phi}(\omega)=\left[\begin{array}{c:c}
\left(\mathbf{S}^{*}(\omega) \mathbf{S}^{T}(\omega)\right) \otimes\left(\mathbf{I}_{J} \otimes\left(\mathbf{z}_{Q}(\omega) \mathbf{z}_{Q}^{H}(\omega)\right)\right) & \operatorname{vec}\left(\mathbf{Y}(\omega) \mathbf{S}^{H}(\omega)\right) \otimes\left(\mathbf{z}_{Q}(\omega) \mathbf{z}_{P}^{H}(\omega)\right) \\
\hdashline \operatorname{vec}\left(\mathbf{Y}(\omega) \mathbf{S}^{H}(\omega)\right)^{H} \otimes\left(\mathbf{z}_{P}(\omega) \mathbf{z}_{Q}^{H}(\omega)\right) & \mathbf{Y}^{H}(\omega) \mathbf{Y}(\omega) \mathbf{z}_{P}(\omega) \mathbf{z}_{P}^{H}(\omega)
\end{array}\right]
$$

of the wave equation is not taken into account. In this paper, we will adopt the frequency domain identification algorithm proposed in Verboven et al. (2004) as the stateof-the-art algorithm. In the frequency domain, the data model corresponding to (5)-(8) can be written as

$$
\mathbf{Y}(\omega)=\frac{\mathcal{B}(\omega)}{A(\omega)} \mathbf{S}(\omega)+\mathbf{V}(\omega)
$$

where $\omega=2 \pi f T_{s}$ denotes radial frequency,

$$
\begin{aligned}
\mathbf{S}(\omega) & =\left[\begin{array}{lll}
S_{1}(\omega) & \ldots S_{M}(\omega)
\end{array}\right]^{T} \\
\mathbf{V}(\omega) & =\left[\begin{array}{llll}
V_{1}(\omega) & \ldots V_{J}(\omega)
\end{array}\right]^{T} \\
\mathbf{Y}(\omega) & =\left[\begin{array}{lll}
Y_{1}(\omega) & \ldots Y_{J}(\omega)
\end{array}\right]^{T}
\end{aligned}
$$

contain the $N$-point discrete Fourier transform (DFT) samples of the source signals, measurement noise, and observed signals, $A(\omega)$ represents the pole-zero model denominator frequency response, and

$$
\mathcal{B}(\omega)=\left[\begin{array}{ccc}
B_{11}(\omega) & \ldots & B_{1 M}(\omega) \\
\vdots & \ddots & \vdots \\
B_{J 1}(\omega) & \ldots & B_{J M}(\omega)
\end{array}\right]
$$

contains the pole-zero model numerator frequency responses for the different source-observer combinations.

By defining the equation error vector related to (13) as

$$
\mathbf{E}(\omega, \boldsymbol{\theta})=\boldsymbol{B}(\omega) \mathbf{S}(\omega)-A(\omega) \mathbf{Y}(\omega)
$$

a least squares (LS) criterion for the estimation of the parameter vector $\boldsymbol{\theta}$ can be obtained as

$$
\min _{\boldsymbol{\theta}} \sum_{\omega} \mathbf{E}^{H}(\omega, \boldsymbol{\theta}) \mathbf{E}(\omega, \boldsymbol{\theta})
$$

where the summation is executed over the DFT frequencies $\omega=0,1 / N, \ldots,(N-1) / N$, and $(\cdot)^{H}$ denotes the Hermitian transposition operator. The LS criterion (19) can be rewritten as a quadratic program $(\mathrm{QP})$,

$$
\begin{array}{ll}
\min _{\boldsymbol{\theta}} & \boldsymbol{\theta}^{T}\left(\sum_{\omega} \boldsymbol{\Phi}(\omega)\right) \boldsymbol{\theta} \\
\text { s. t. } & a_{0}=1
\end{array}
$$

with $\boldsymbol{\Phi}(\omega)$ defined in (12) at the top of the page, where $\mathbf{I}_{J}$ represents the $J \times J$ identity matrix, $(\cdot)^{*}$ denotes the complex conjugation operator, $\operatorname{vec}(\cdot)$ is the matrix vectorization operator, $\otimes$ denotes the Kronecker product, and the complex sinusoidal vectors are defined as

$$
\begin{aligned}
& \mathbf{z}_{Q}(\omega)=\left[\begin{array}{llll}
1 & e^{j \omega} & \ldots & e^{j Q \omega}
\end{array}\right]^{T} \\
& \mathbf{z}_{P}(\omega)=\left[\begin{array}{llll}
1 & e^{j \omega} & \ldots & e^{j P \omega}
\end{array}\right]^{T} .
\end{aligned}
$$

\section{FINITE ELEMENT METHOD FOR COMMON-DENOMINATOR POLE-ZERO MODELS}

We will now derive a set of linear equations in the polezero model parameters, that are valid if the MIMO-LTI system is indeed governed by the wave equation in (1). We can eliminate the time variable and the partial time derivative from the wave equation by taking the discrete Fourier transform of (1) after temporal sampling, which results in the Helmholtz equation

$$
\nabla^{2} U(\mathbf{r}, \omega)+k^{2} U(\mathbf{r}, \omega)=S(\mathbf{r}, \omega)
$$

where $k=\omega / c$ represents the wave number. As mentioned earlier, we consider the source function to consist of $M$ point source contributions, i.e.,

$$
S(\mathbf{r}, \omega)=\sum_{m=1}^{M} S_{m}(\omega) \delta\left(\mathbf{r}-\mathbf{r}_{m}\right) .
$$

By substituting (25) in (24) and dividing both sides by $S_{m}(\omega), m=1, \ldots, M$ we obtain a set of $M$ equations

$$
\left\{\begin{array}{c}
\nabla^{2} H\left(\mathbf{r}, \mathbf{r}_{1}, \omega\right)+k^{2} H\left(\mathbf{r}, \mathbf{r}_{1}, \omega\right)=\sum_{m=1}^{M} \frac{S_{m}(\omega)}{S_{1}(\omega)} \delta\left(\mathbf{r}-\mathbf{r}_{m}\right) \\
\vdots \\
\vdots \\
\nabla^{2} H\left(\mathbf{r}, \mathbf{r}_{M}, \omega\right)+k^{2} H\left(\mathbf{r}, \mathbf{r}_{M}, \omega\right)=\sum_{m=1}^{M} \frac{S_{m}(\omega)}{S_{M}(\omega)} \delta\left(\mathbf{r}-\mathbf{r}_{m}\right)
\end{array}\right.
$$

where the frequency-domain Green's function $H\left(\mathbf{r}, \mathbf{r}_{m}, \omega\right)$, $m=1, \ldots, M$, corresponds to the frequency response of the discrete-time system defined in (4) for $\mathbf{r}=$ $\overline{\mathbf{r}}_{j}, j=1, \ldots, J$. We can hence substitute the commondenominator pole-zero model for $H\left(\mathbf{r}, \mathbf{r}_{m}, \omega\right)$ in $(26)$, and bring the common denominator (which is independent of r) to the right-hand side, i.e.,

$$
\left\{\begin{array}{r}
\nabla^{2} B\left(\mathbf{r}, \mathbf{r}_{m}, \omega\right)+k^{2} B\left(\mathbf{r}, \mathbf{r}_{m}, \omega\right)=A(\omega) \sum_{l=1}^{M} \frac{S_{l}(\omega)}{S_{m}(\omega)} \delta\left(\mathbf{r}-\mathbf{r}_{l}\right), \\
m=1, \ldots, M .
\end{array}\right.
$$

where we have used a more compact notation to denote a set of $M$ equations. Note that we have deliberately not restricted the observer position $\mathbf{r}$ in (27) to the discrete set of positions $\overline{\mathbf{r}}_{j}$ defined earlier. Instead, we consider the numerator frequency response $B\left(\mathbf{r}, \mathbf{r}_{m}, \omega\right)$ to be a continuous function of $\mathbf{r}$. This function can be approximated in a finite-dimensional subspace by discretizing the spatial domain $\Omega$ using a 3-D grid defined by the points $\overline{\mathbf{r}}_{k}, k=1, \ldots, K$ with $K \geq J$ (and typically $K \gg J$ ), which includes the observer positions,

$$
B\left(\mathbf{r}, \mathbf{r}_{m}, \omega\right) \approx \sum_{k=1}^{K} B\left(\overline{\mathbf{r}}_{k}, \mathbf{r}_{m}, \omega\right) \phi_{k}(\mathbf{r})
$$

Here, the subspace basis functions are chosen to be piecewise linear functions satisfying $\phi_{i}\left(\overline{\mathbf{r}}_{k}\right)=\delta(i-k), i=$ $1, \ldots, K$. In particular, the basis functions are defined on a 3-D triangulation of the spatial domain $\Omega$, where the $k$ th basis function is made up of linear (non-zero slope) segments along the line segments between the point $\overline{\mathbf{r}}_{k}$ and all the points with which point $\overline{\mathbf{r}}_{k}$ shares a tetrahedron edge, and zero-valued segments elsewhere. We can then rewrite the set of Helmholtz equations in (27) as a set of linear equations in $B\left(\overline{\mathbf{r}}_{k}, \mathbf{r}_{m}, \omega\right)$ by making use of the FEM, see Brenner and Scott (2008). In a nutshell, the FEM consists in converting the partial differential equation (PDE) in (27) to its weak formulation, performing integration by parts to relax the differentiability requirements on the subspace basis functions, and enforcing the subspace approximation error induced by (28) to be orthogonal to this subspace. The set of $M$ PDEs in (27) can then be 
expanded to a set of $M K$ linear equations, also known as the Galerkin equations,

$$
\left\{\left(\mathbf{K}-k^{2} \mathbf{L}\right) \boldsymbol{\beta}_{m}(\omega)=-A(\omega) \sum_{\substack{l=1 \\ m=1, \ldots, M}}^{\frac{S_{l}(\omega)}{S_{m}(\omega)} \boldsymbol{\psi}_{l},},\right.
$$

Here, the $K \times K$ matrices $\mathbf{K}$ and $\mathbf{L}$ denote the FEM stiffness and mass matrices, defined as

$$
\begin{aligned}
{[\mathbf{K}]_{i j} } & =\int_{\Omega} \nabla \phi_{j}(\mathbf{r}) \cdot \nabla \phi_{i}(\mathbf{r}) d \mathbf{r} \\
{[\mathbf{L}]_{i j} } & =\int_{\Omega} \phi_{j}(\mathbf{r}) \phi_{i}(\mathbf{r}) d \mathbf{r}
\end{aligned}
$$

and the $K \times 1$ vector $\boldsymbol{\beta}_{m}(\omega)$ contains the spatial samples of the function $B\left(\mathbf{r}, \mathbf{r}_{m}, \omega\right)$ as defined by (28), i.e.,

$$
\boldsymbol{\beta}_{m}(\omega)=\left[B\left(\overline{\mathbf{r}}_{1}, \mathbf{r}_{m}, \omega\right) \ldots B\left(\overline{\mathbf{r}}_{K}, \mathbf{r}_{m}, \omega\right)\right]^{T} .
$$

The $K \times 1$ vectors $\psi_{l}, l=1, \ldots, M$ on the right-hand side of the Galerkin system in (29) contain the barycentric coordinates of the point sources, obtained by projecting the spatial unit-impulse functions $\delta\left(\mathbf{r}-\mathbf{r}_{l}\right)$ onto the chosen subspace basis, i.e.,

$$
\left[\boldsymbol{\psi}_{l}\right]_{i}=\int_{\Omega} \delta\left(\mathbf{r}-\mathbf{r}_{l}\right) \phi_{i}(\mathbf{r}) d \mathbf{r} .
$$

Each vector $\psi_{l}$ has only $1,2,3$, or 4 non-zero elements, depending on whether the $m$ th point source is located in a vertex, on an edge, on a face, or in the interior of a tetrahedron of the FEM mesh. We can write (29) in a more compact notation by defining $M \times 1$ vectors $\chi_{m}, m=1, \ldots, M$, containing the source spectrum ratios,

$$
\chi_{m}(\omega)=\left[\frac{S_{1}(\omega)}{S_{m}(\omega)} \cdots \frac{S_{M}(\omega)}{S_{m}(\omega)}\right]^{T}, m=1, \ldots, M
$$

and the $K \times M$ matrix

$$
\boldsymbol{\Psi}=\left[\boldsymbol{\psi}_{1} \ldots \boldsymbol{\psi}_{M}\right]
$$

such that

$$
\left\{\begin{array}{r}
\left(\mathbf{K}-k^{2} \mathbf{L}\right) \boldsymbol{\beta}_{m}(\omega)=-A(\omega) \boldsymbol{\Psi} \boldsymbol{\chi}_{m}(\omega), \\
m=1, \ldots, M .
\end{array}\right.
$$

Finally, we can write the Galerkin equations as a function of the model parameters of the common-denominator polezero model defined in (5) as follows. Define the $K(Q+1) \times 1$ numerator parameter vector, for $m=1, \ldots, M$,

$$
\underline{\mathbf{b}}_{m}=\left[b_{0}\left(\overline{\mathbf{r}}_{1}, \mathbf{r}_{m}\right) \ldots b_{Q}\left(\overline{\mathbf{r}}_{1}, \mathbf{r}_{m}\right) \ldots b_{0}\left(\overline{\mathbf{r}}_{K}, \mathbf{r}_{m}\right) \ldots b_{Q}\left(\overline{\mathbf{r}}_{K}, \mathbf{r}_{m}\right)\right]^{T}
$$

and recall the $(P+1) \times 1$ denominator parameter vector definition in (11). Note that only the first $J(Q+1)$ coefficients of the numerator parameter vector (corresponding to the elements of the numerator parameter vector $\mathbf{b}_{m}$ defined in (10)) are of explicit interest, while the other coefficients have been introduced for constructing the FEM approximation of the continuous-space function $B\left(\mathbf{r}, \mathbf{r}_{m}, \omega\right)$. By using the above parameter vector definitions, and recalling the definitions of the complex sinusoidal vectors in (22)-(23), we can rewrite the Galerkin system in (36) as follows,

$$
\left\{\left(\mathbf{K}-k^{2} \mathbf{L}\right)\left(\mathbf{I}_{K} \otimes \mathbf{z}_{Q}^{H}(\omega)\right) \underline{\mathbf{b}}_{m}=-\mathbf{\Psi} \chi_{m}(\omega) \mathbf{z}_{P}^{H}(\omega) \mathbf{a},\right.
$$$$
m=1, \ldots, M(38)
$$

or equivalently

$$
\underbrace{\left[\begin{array}{ccccc}
\mathbf{M}(\omega) & \mathbf{0} & \ldots & \mathbf{0} & \boldsymbol{\Psi} \boldsymbol{\chi}_{1}(\omega) \mathbf{z}_{P}^{H}(\omega) \\
\mathbf{0} & \mathbf{M}(\omega) & \ldots & \mathbf{0} & \boldsymbol{\Psi} \chi_{2}(\omega) \mathbf{z}_{P}^{H}(\omega) \\
\vdots & \vdots & \ddots & \vdots & \vdots \\
\mathbf{0} & \mathbf{0} & \ldots & \mathbf{M}(\omega) & \boldsymbol{\Psi} \boldsymbol{\chi}_{M}(\omega) \mathbf{z}_{P}^{H}(\omega)
\end{array}\right]}_{\boldsymbol{\Xi}(\omega)} \underbrace{\left[\begin{array}{c}
\underline{\mathbf{b}}_{1} \\
\underline{\mathbf{b}}_{2} \\
\vdots \\
\underline{\mathbf{b}}_{M} \\
\mathbf{a}
\end{array}\right]}_{\underline{\boldsymbol{\theta}}}=\mathbf{0}
$$

Here, $\mathbf{M}(\omega)=\left(\mathbf{K}-k^{2} \mathbf{L}\right)\left(\mathbf{I}_{K} \otimes \mathbf{z}_{Q}^{H}(\omega)\right)$, and $\mathbf{0}$ represents a zero vector or matrix of appropriate dimensions.

A few remarks are in place here. First, the Galerkin system in (39) is always underdetermined. However, we can straightforwardly increase the number of equations by considering (39) for $L$ different radial frequencies $\omega_{l}, l=$ $1, \ldots, L$, without increasing the dimension of the parameter vector. It suffices to choose $L \geq Q+1+(P+1) /(M K)$ to obtain a square or overdetermined system of equations. Second, a well-known and attractive property of the FEM is that the stiffness and mass matrices $\mathbf{K}$ and $\mathbf{L}$, as well as the point source positioning matrix $\boldsymbol{\Psi}$, are highly sparse and structured. Consequently, the system of equations in (39) can typically be solved with a linear complexity. Third, we should stress that the accuracy of the FEM approximation relies heavily on the quality of the mesh, which is why we cannot just set $K=J$ and define the FEM mesh using only the observer positions $\overline{\mathbf{r}}_{j}, j=1, \ldots, J$. In particular, a sufficiently large number of mesh points is needed to achieve a good spatial resolution and nearuniformity of the tetrahedra defined in the triangulation.

\section{PROPOSED IDENTIFICATION APPROACH}

The proposed identification approach is aimed at blending measured information in the data set with structural information obtained from the wave equation, and results from the integration of the Galerkin equations (39) in the QP (20)-(21). One way to achieve this integration is to apply the field estimation framework proposed in van Waterschoot and Leus (2011), where an optimization problem is defined in which a LS data-based objective function is minimized subject to the Galerkin equations. If we apply this framework to the problem considered here, we end up with a large-scale equality-constrained QP,

$$
\begin{array}{cc}
\min _{\underline{\boldsymbol{\theta}}} & \underline{\boldsymbol{\theta}}^{T} \mathbf{C}^{T}\left(\sum_{\omega} \boldsymbol{\Phi}(\omega)\right) \mathbf{C} \underline{\boldsymbol{\theta}} \\
\text { s.t. } & \left\{\begin{array}{c}
\boldsymbol{\Xi}\left(\omega_{1}\right) \underline{\boldsymbol{\theta}}=\mathbf{0} \\
\vdots \\
\boldsymbol{\Xi}\left(\omega_{L}\right) \underline{\boldsymbol{\theta}}=\mathbf{0} \\
a_{0}=1
\end{array}\right.
\end{array}
$$

Here, the $[J(Q+1)+P+1] \times[K(Q+1)+P+1]$ selection matrix $\mathbf{C}$ is defined such that

$$
\mathbf{C} \underline{\boldsymbol{\theta}}=\boldsymbol{\theta} .
$$

Compared to the state-of-the-art identification approach exemplified by the QP in (20)-(21), $L M K$ additional equality constraints have been included in (41). These equality constraints allow to impose structural information at a number of frequencies $\omega_{1}, \ldots, \omega_{L}$, thus increasing the model accuracy at these particular frequencies. The number of frequencies $L$ at which the Galerkin equations 
are imposed in (41) should satisfy that $L \leq Q+(P+$ 1)/ $(M K)$, as otherwise an infeasible QP may be obtained.

Up till now we have assumed that all quantities required in the computation of the matrices $\boldsymbol{\Xi}(\omega)$ and $\mathbf{C}$ are available. More particularly, $\boldsymbol{\Xi}(\omega)$ relies on the geometry of the FEM mesh (through $\mathbf{K}, \mathbf{L}$, and $\boldsymbol{\Psi}$ ), on the source spectrum ratios (through $\chi_{m}(\omega)$ ), and on the point source positions (through $\boldsymbol{\Psi}$ ), while $\mathbf{C}$ depends on the observer positions. In a typical identification experiment, the source spectrum ratios and the observer positions are indeed known, while the FEM mesh is known by construction. However, in many applications, the point source positions are unknown and so the point source positioning matrix $\boldsymbol{\Psi}$ cannot be straightforwardly computed. Nevertheless, we will show that if a preliminary estimate $\hat{\mathbf{a}}$ of the pole-zero model denominator parameter vector is available (e.g., by using the state-of-the-art data-based identification approach outlined in Section 2.2), the point source positioning matrix $\boldsymbol{\Psi}$ can be estimated by exploiting its particular structure and sparsity. To this end, we first rewrite (36) as

$$
\begin{array}{r}
\left(\mathbf{K}-k^{2} \mathbf{L}\right) \boldsymbol{\beta}_{m}(\omega)=-\left(\boldsymbol{\chi}_{m}^{T}(\omega) \otimes \hat{A}(\omega) \mathbf{I}_{K}\right) \overbrace{\operatorname{vec}(\mathbf{\Psi})}^{\boldsymbol{\psi}}, \\
m=1, \ldots, M(43)
\end{array}
$$

or equivalently

$$
\underbrace{\left[\begin{array}{ccccc}
\mathbf{M}(\omega) & \mathbf{0} & \ldots & \mathbf{0} & \boldsymbol{\chi}_{1}^{T}(\omega) \otimes \hat{A}(\omega) \mathbf{I}_{K} \\
\mathbf{0} & \mathbf{M}(\omega) & \ldots & \mathbf{0} & \boldsymbol{\chi}_{2}^{T}(\omega) \otimes \hat{A}(\omega) \mathbf{I}_{K} \\
\vdots & \vdots & \ddots & \vdots & \vdots \\
\mathbf{0} & \mathbf{0} & \ldots & \mathbf{M}(\omega) & \boldsymbol{\chi}_{M}^{T}(\omega) \otimes \hat{A}(\omega) \mathbf{I}_{K}
\end{array}\right]}_{\boldsymbol{\Delta}(\hat{\mathbf{a}}, \omega)} \underbrace{\left[\begin{array}{c}
\underline{\mathbf{b}}_{1} \\
\underline{\mathbf{b}}_{2} \\
\vdots \\
\underline{\mathbf{b}}_{M} \\
\boldsymbol{\psi}
\end{array}\right]}_{\boldsymbol{\zeta}}=\mathbf{0 .}
$$

The data term in (40) can also be rewritten as a function of $\boldsymbol{\zeta}$, by partitioning the matrix $\left(\sum_{\omega} \boldsymbol{\Phi}(\omega)\right)^{1 / 2} \mathbf{C} \triangleq\left[\boldsymbol{\Gamma}_{L} \mid \boldsymbol{\Gamma}_{R}\right]$ such that

$$
\left(\sum_{\omega} \boldsymbol{\Phi}(\omega)\right)^{1 / 2} \mathbf{C} \underline{\boldsymbol{\theta}}=\boldsymbol{\Gamma}_{L} \overbrace{\left[\mathbf{I}_{M K(Q+1)} \mathbf{0}\right]}^{\mathbf{F}} \boldsymbol{\zeta}+\boldsymbol{\Gamma}_{R} \mathbf{a} .
$$

Again, data information and structural information can be combined into a single convex optimization problem in which the point source positioning matrix $\boldsymbol{\Psi}$ is estimated alongside the pole-zero model numerator coefficients, i.e.,

$$
\begin{aligned}
\min _{\boldsymbol{\zeta}} & \left\|\boldsymbol{\Gamma}_{L} \mathbf{F} \boldsymbol{\zeta}+\boldsymbol{\Gamma}_{R} \hat{\mathbf{a}}\right\|_{2}+\|\boldsymbol{\Delta}(\hat{\mathbf{a}}, \omega) \boldsymbol{\zeta}\|_{2}+\lambda\|\boldsymbol{\psi}\|_{1} \\
\text { s. t. } & \left\{\begin{aligned}
\left(\mathbf{I}_{M} \otimes \mathbf{1}_{1 \times K}\right) \boldsymbol{\psi} & =\mathbf{1}_{M \times 1} \\
\boldsymbol{\psi} & \geq \mathbf{0}
\end{aligned}\right.
\end{aligned}
$$

with 1 a vector of all ones. In this optimization problem, the sparsity of $\boldsymbol{\Psi}$ is exploited by including an $\ell 1-$ regularization term in (46), while the non-negativity and the property of columns summing to one, are enforced in the (in)equality constraints (47).

\section{SIMULATION RESULTS}

We provide a simulation example, in which the proposed identification approach is compared to the state-of-the-art approach for the case of indoor acoustic wave propagation $(c=344 \mathrm{~m} / \mathrm{s})$. We consider a rectangular room of $8 \times$ $6 \times 4 \mathrm{~m}$, with $M=3$ sources and $J=5$ sensors positioned as shown in Fig. 1. The Green's functions

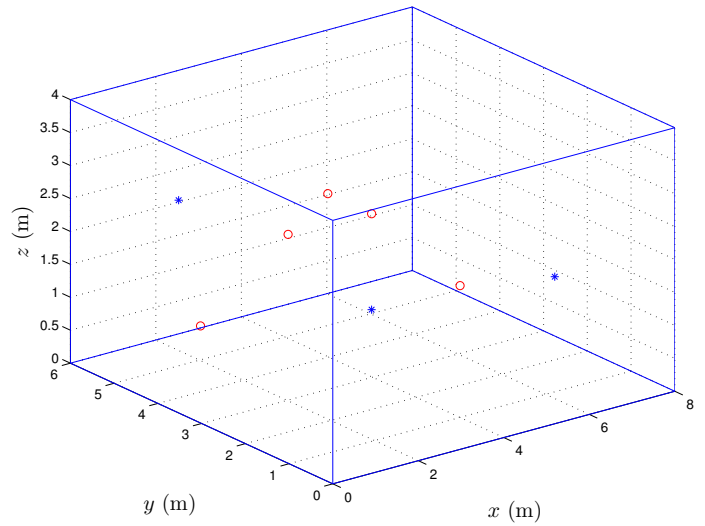

Fig. 1. Simulation scenario: rectangular room $(8 \times 6 \times 4$ m) with $M=3$ sources (blue $*$ ) and $J=5$ sensors (red o).

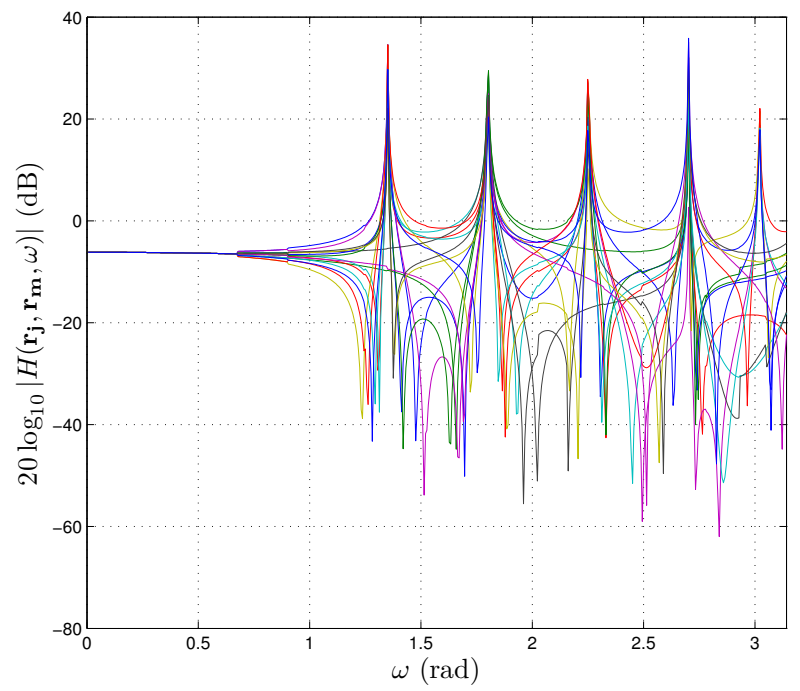

Fig. 2. Frequency magnitude responses of the Green's functions related to the different source-observer combinations.

related to the source and observer positions have been simulated using the assumed modes solution to the wave equation, see Gustafsson et al. (2000), truncated to a duration of $10 \mathrm{~s}$, sampled at $f_{s}=100 \mathrm{~Hz}$, and low-pass filtered to suppress the "cavity mode" at DC. The resulting frequency magnitude responses $20 \log _{10}\left|H\left(\overline{\mathbf{r}}_{j}, \mathbf{r}_{m}, \omega\right)\right|$ for $m=1, \ldots, M, j=1, \ldots, J$ are plotted in Fig. 3. The common resonances can be clearly observed.

The data set was generated as follows: the $M$ source signals were obtained by filtering $M$ Gaussian white noise signals with $M$ different all-pole filters (first-order lowpass, second-order band-pass, and first-order high-pass for $m=1,2,3$, respectively). The observed signals were obtained by filtering the source signals with the simulated Green's functions and adding Gaussian white noise at a $0 \mathrm{~dB}$ signal-to-noise ratio (SNR). The FEM mesh was generated by performing a 3-D Delaunay triangulation on a set of 315 regularly spaced grid points separated by 1 $\mathrm{m}$ in each dimension. The resulting FEM mesh consists of 1152 elements, and is shown in Fig. 3. 


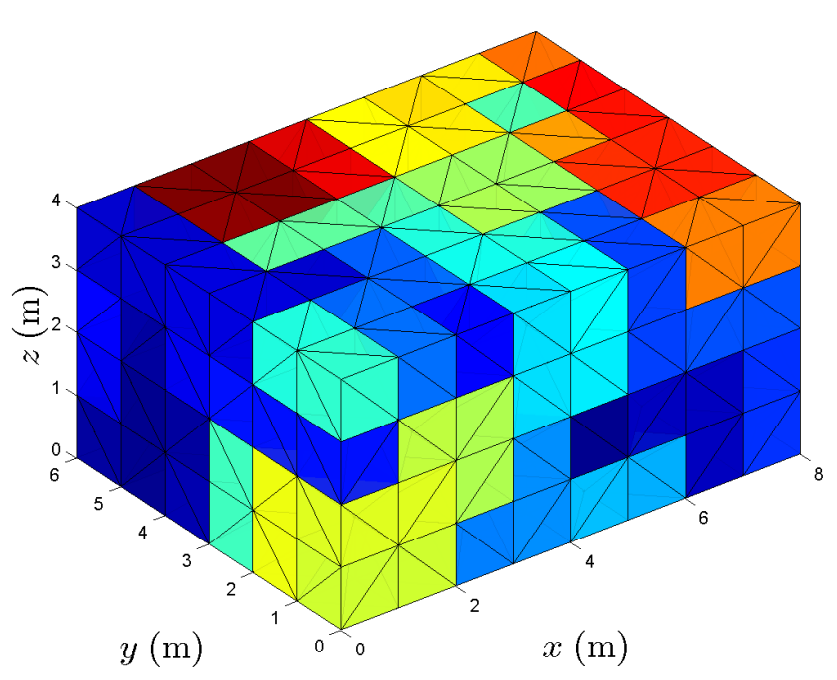

Fig. 3. Visualization of the tetrahedral FEM mesh.

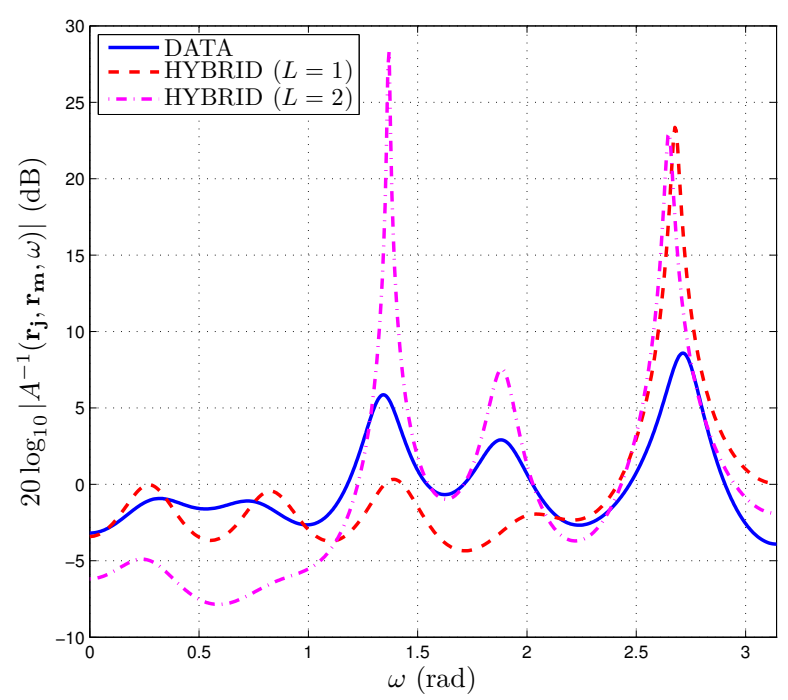

Fig. 4. Results with exact source positioning matrix.

We evaluate the capability of the data-based ("DATA") and proposed ("HYBRID") identification approaches to capture the resonant behavior of the wave propagation, by inspecting the pole-zero model inverse denominator frequency magnitude response $20 \log _{10}\left|A^{-1}\left(\overline{\mathbf{r}}_{j}, \mathbf{r}_{m}, \omega\right)\right|$. The pole-zero model orders are set to $Q=P=12$. The proposed approach is evaluated with the Galerkin equality constraints imposed at $L=1$ frequency and $L=2$ frequencies. These frequencies are chosen to correspond to the 4 th and 1st resonance frequency of the Green's functions, respectively, i.e., $\omega_{1}=2.7018 \mathrm{rad}$ and $\omega_{2}=1.3509 \mathrm{rad}$.

Fig. 4 shows the results for the case when the point source positions are exactly known, and hence (40)-(41) can be directly solved. It is clearly observed that by imposing the Galerkin equality constraints at a certain frequency, the resonant behavior at that particular frequency is identified much more accurately compared to the case when only measurement information is used.

Finally, Fig. 5 shows the results for the case when the point source positions are unknown, and the point source positioning matrix $\boldsymbol{\Psi}$ is estimated using the sparse ap-

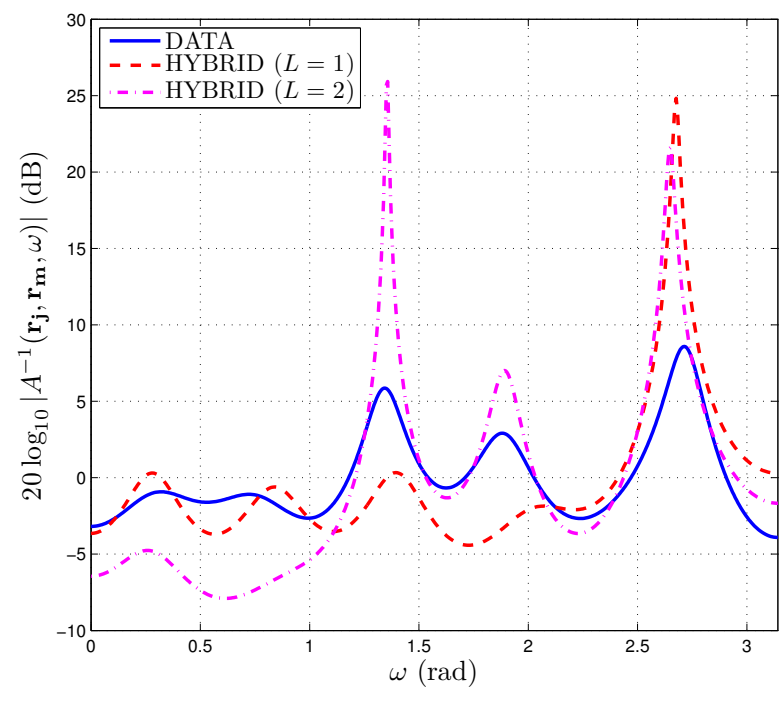

Fig. 5. Results with estimated source positioning matrix. proximation algorithm (46)-(47) prior to executing the hybrid identification algorithm (40)-(41). The resulting identification performance is seen to be comparable to the case when exact knowledge of the point source positions is assumed.

\section{REFERENCES}

Brenner, S.C. and Scott, L.R. (2008). The mathematical theory of finite element methods. Springer, New York.

Gustafsson, T., Vance, J., Pota, H.R., Rao, B.D., and Trivedi, M.M. (2000). Estimation of acoustical room transfer functions. In Proc. 39th IEEE Conf. Decision Control (CDC '00), 5184-5189. Sydney, Australia.

Haneda, Y., Makino, S., and Kaneda, Y. (1994). Common acoustical pole and zero modeling of room transfer functions. IEEE Trans. Speech Audio Process., 2(2), $320-328$.

Hikichi, T. and Miyoshi, M. (2004). Blind algorithm for calculating common poles based on linear prediction. In Proc. 2004 IEEE Int. Conf. Acoust., Speech, Signal Process. (ICASSP '04), volume 4, 89-92. Montreal, Quebec, Canada.

Kuttruff, H. (2009). Room Acoustics. Spon Press, London.

Rolain, Y., Vandersteen, G., and Schoukens, J. (1998). Best conditioned common denominator transfer function matrix estimation in the frequency domain. In Proc. 37th IEEE Conf. Decision Control (CDC '98), 39383939. Tampa, Florida, USA.

Stoica, P. and Jansson, M. (2000). MIMO system identification: State-space and subspace approximations versus transfer function and instrumental variables. IEEE Trans. Signal Process., 48(11), 3087-3099.

van Waterschoot, T. and Leus, G. (2011). Static field estimation using a wireless sensor network based on the finite element method. In Proc. Int. Workshop Comput. Adv. Multi-Sensor Adaptive Process. (CAMSAP '11), 369-372. San Juan, PR, USA.

Verboven, P., Guillaume, P., Cauberghe, B., Vanlanduit, S., and Parloo, E. (2004). Modal parameter estimation from input-output Fourier data using frequencydomain maximum likelihood identification. J. Sound Vib., 276(3-5), 957-979. 